\title{
Problem of Energy and the Second Law of Thermodynamics
}

\section{George Vasilevich Skornyakov}

Ioffe Institute, St.-Petersburg, Russia

\section{Email address:}

skorn@ioffe.ru

\section{To cite this article:}

George Vasilevich Skornyakov. Problem of Energy and the Second Law of Thermodynamics. International Journal of Energy and Power Engineering. Vol. 8, No. 1, 2019, pp. 1-3. doi: 10.11648/j.ijepe.20190801.11

Received: December 26, 2018; Accepted: March 2, 2019; Published: March 20, 2019

\begin{abstract}
Receipt of heat in a thermodynamic system is defined by a Pfaffian form. For the heat-insulated systems it is equal to zero. Cooling of such system as a result of cyclic change of external parameters is usually considered to be impossible. It is offered to carry out full transformation of heat of the environment to work by means of thermally heterogeneous converter containing two gases with different thermal capacities divided by a mobile heatimpenetrable partition. As a result of cyclic change of external parameters along with production of work one gas is cooled, and another - heats up. The possibility of this process is caused by the fact that the entropy of a multiple parameter system cannot be generally defined.
\end{abstract}

Keywords: Energy, Entropy, Laws of Thermodynamics

\section{Introduction}

The last years the increasing attention is paid to renewable energy resources and different use of natural non-equilibriums in the energetic purposes. Visitors to "roaring the fortieth" and neighbors of Niagara will hardly be sometime anxious with search of necessary power sources. But not everywhere such power luxury is available and to not everyone. And here heatand-power transformation comes to the rescue.

In heat-and-power transformer (converter) such "favor of the Nature" is presented by the heater and the cooler. This nonequilibrium serves as premises of production of work by the transformer. Energy necessary for this purpose is provided by the energy carrier (gas, oil, coal, firewood, etc.), But not less essential role of oxygen of the atmosphere in production of work is usually ignored. (A case of nuclear power is not considered.) Not only the role of the atmosphere in heat-and-power transformer, the benefit for it, is ignored at least so far, it is not necessary to pay, but also the fact of our absorption in it. The Second Law of thermodynamics in his standard understanding gets in the way of use of this almost inexhaustible energy [1-7].

\section{Converter Role in Transformation}

Heat transformer plays only a role of transfer function and it is always considered being in thermal equilibrium. The one-orientation of the processes of heat conductivity providing transfer of heat from a hot body to a cold one created illusion of impossibility of other mechanisms of energy exchange between different parts of the transformer. But for the possibiliry to speak about its parts, it should have at least two external parameters. So concerning oneparameter systems the developed ideas of the nature of thermal processes save their force. In relation to multiple parameter systems they are fair only in cases of quite integrability of their Pfaffian form.

\section{Thermally Heterogeneous Converter}

Brigt example, and besides long ago known, of the nonintegrable system is two gases with different heat capacity divided by a mobile heat impenetrable partition. Existence of such partition providing equality of pressure of gases, but not their temperatures, opens possibility during process of conversion of heat to work of thermal heterogeneity in the transformer. As a result of cyclic process one part of the transformer can heat up, and another to be cooled. This non- uniformity arising in process also provides a possibility of full conversion of heat to work as a result of cooling of the environment.

The possibility of cooling of a heat-insulated multiple 
parameter system or its part as a result of cyclic process of change of external parameters radically changes setting of a power problem. Energy resources of the atmosphere of Earth, without telling about oceans, more than enough for satisfaction of ours needs in the near future and in general for all the time of our existence. The imaginary prohibition on their use which is allegedly imposed by laws of thermodynamics is generation of the misunderstanding caused by the data of energy exchange between the contacting parts of a system only to heat conductivity. The senseless heap of the statements based on this false thesis also makes contents of the known article of C. Caratheodory and its "explanations" by M. Born [8 - 9]. They possessed very vague ideas of the nature of thermal processes. So Caratheodory wrote: "... at contact of bodies of various temperature heat always passes from warmer to colder body, on the contrary - never". But on it all acoustics keeps!

The exceptional case was built in the absolute. Both authors managed even not to mention a name of G. F. Frobenius, who long before them established the set condition of quite integrability of the Pfaff equations, defining existence of entropy at any number of variables. For a case of three independent variables these conditions were known still to L. Euler. Ugly symbiosis of ignorance and a credulity provided a wide use and also long and steady existence of these delusions.

\section{Conditional Entropy}

The impossibility of existence of entropy itself as a function of thermodynamic variables generally for multiple parameter systems does not exclude application for their research of much more strict methods, than direct transfer of the terminology which developed for the probability theory in thermodynamics. In case of gases in their basis is the principle of maximum entropy. At first sight it can seem a tautology. Really, how properties of nonexistent value can define the behavior of a real system?

Let's explain it on a specific example of two different gases divided by a mobile heat impenetrable partition. In lack of heat exchange with the external environment at change of external parameters each gas changes its volume in adiabatic conditions (saving its entropy). Existence of a mobile heat impenetrable partition provides integrity of a system and energy exchange between its parts and also possibility itself in them to the thermal heterogeneity caused by the difference of temperatures of parts of a system.

Though the entropy of a multiple parameter system generally also does not exist, it is possible to enter concepts of conditional entropy which number is equal to number of external parameters. At the change of any one external parameter (in the conditions of the invariance of the others) the conditional entropy of a system does not change, but transition to change of another one is connected with the redefinition of conditional entropy. Need of such redefinition practically for any cyclic process also causes impossibility of existence of entropy of multiple parameter systems. As for entropy of parts of a system, their sum, though remains, in general has no relation to entropy of a system. The thermodynamic entropy, unlike entropy in probability theory, is defined only for equilibrium states and quasistatic processes. The considered system provides only equality of pressure, while gas temperatures can be different.

\section{Processes in Thermally Heterogeneous Systems}

Addition only of one independent variable cardinally changes properties of a system and opens a possibility of implementation of essentially new processes. There is possible a cyclic process of conversion of the heat into work, and work into heat in a heat-insulated system.

If in an initial state the system was in thermal balance with the thermostat (external environment), the cycle of change of external parameters which is carried out in the conditions of heat insulation will decrease temperature of one gas and will increase temperature of another. Recovery of thermal equilibrium with the thermostat will renew an initial state.

Obviously, cyclic changes of any one external parameter do not lead to change of a system. But if after shift of the right piston to the right left too shift a little on the left, end a cycle will lead to small production of work at any shifts of the right piston and small shifts of left. Along with it the partition will a little be displaced to the right, the left gas will extend and will be cooled, and right - will contract and will heat up. The work of the left piston as the size of the second order of trifle, is possible to neglect. Restoration of thermal balance with the thermostat and repetition of a cycle in the conditions of heat insulation will be led to gradual transition of heat to work contrary to the standard formulations of the Second Law.

So insignificant production of work for a cycle will hardly be of practical interest. But at big shifts of the right and left pistons it is easy to achieve almost full transition of heat to work for one cycle. If gas with bigger thermal capacity is located on the right, in an initial state, heat is concentrated generally in the right gas, especially if the initial volume of the left gas is much less than the volume of right. In process of shift of the right piston to the right both gases are cooled, and left more. The heatimpenetrable piston dividing a system moves to the right. At rather big shift of the right piston almost all heat turns into work. Pressure and temperatures of both gases tends to zero.

As a result of shift of the left piston on the left at the same size, as shift of right, will proceed pressure decrease and gas temperatures, but no work is performed in view of extremely low pressure in a system. The dividing piston will move on the left.

Return of the right piston to a starting position happens almost without work expenses in view of extremely low pressure in a system, and is followed by further shift of the dividing piston on the left. In process of the movement of the left piston in a starting position pressure grows in a system, 
but cannot exceed initial as the right piston already made work. The dividing piston moves to the right, but does not reach a starting position. As a result the left gas adiabatically contracts and heats up, and right — extends adiabatically and cooled. The work made by the right piston and heating of the left gas are made due to cooling of the right gas.

\section{Holding of Gases by Gravitational Field}

Mistakes are peculiar often take roots in consciousness contrary to evidence. In gravitational field of Earth not only density, but also temperature of the atmosphere decreases with height [10]. The last circumstance was never taken earlier into account though it is not necessary to be the climber that to be convinced of it. Certainly, the atmosphere is the non-equilibrium object, but the fact of its existence testifies not too big deviations from an equilibrium. Authors [11], proceeding from disappearance of gravitation in process of removal from the planet, come to a conclusion about impossibility of keeping by planets of their atmospheres. According to them the gas atmosphere dissipates though they do not offer any explanation for the fact of existence of the atmospheres at a number of planets. Really, according to a barometric formula it is easy to determine effective "thickness" of the atmosphere. Accounting of dependence of temperature on height does gas pressure drop by much sharper, and "thickness" is more certain [10]. At the maximum height the pressure and temperature reduse to zero. These estimates are fair at considerable excess of radius of the planet over the maximum height of the atmosphere. In relation to planets of the Solar system they are justified in relation to all gases only for huge Jupiter and Saturn which atmospheres contain about ten percent of hydrogen. Venus and Earth cannot hold of light gases (hydrogen and helium), and Mercury is so small that is not able to hold the gas atmosphere at all.

\section{Conclusion}

Not only energy of the atmosphere of Earth, but also its daily variations are so considerable that in comparison with them influence of possible use of its part on ours needs can be neglected. Temperature of the heater is usually maintained by combustion of fuel. Unlike products of combustion of hydrocarbons cyclic process of transformation of heat to work does not change the chemical composition of the atmosphere.

Repetition of a cycle of transformation of heat to work leads to the increasing in a difference of gas temperatures. Thermodynamic equilibrium in each gas is established almost instantly, for times of an order of the relation of the linear sizes of a system to acoustic speed. Growth of a difference of gas temperatures leads to increase in a stream of heat from the heated gas to the cooled one as heat conductivity of a partition though is small, but nevertheless of course exists. It limits converter power.

Need for transportation of fuel disappears as also need of power lines disappears too. In any right place the converter of necessary power working in the autonomous mode can be installed. No problem of energy, its receiving and transportation, exists. The solution of this problem - in the fact of existence of the atmosphere. It comes down not to use of non-equilibrium in the external environment, but to creation of thermal heterogeneity in the converter during the production of a work.

\section{References}

[1] Albert Einstein: Philosopher-Scientist, ed. By P. Schlipp, Evanston, Ill. 1949.

[2] Prigogine I, Science, 1978, vol. 201, No. 4358, pp. 777—785.

[3] Truesdell C., The Tragicomical History of Thermodynamics 1822-1854, N.-Y.; Springer Verlag, 1982, 339 pp.

[4] Fermi E., Thermodynamics, London \& Glasgow, Blackie Ltd., 1938, $160 \mathrm{pp}$.

[5] Second Law of Thermodynamics. Collection of papers (In Russian), M.-L., Gostekhizdat, 1934, 310 pp.

[6] Afanas'eva-Ehrenfest T. A., Zhurn. Prikl. Phys., 1928, vol. 5, parts 3-4, pp. 3-30.

[7] Skornyakov G. V. Techn. Phys. 1996. vol. 66. iss. 1. Pp. 1...11.

[8] Caratheodory C, Math. Ann., 67 (1909), 355-386.

[9] Born M, Phys. Zeit. 22, (1921), 218-224, 249-254, 282286.

[10] Skornyakov G. V., Principles of the theory of thermal processes, LAMBERT Academic Publishing, 2017.

[11] Landau L. D. and Lifshits E. M., Theoretical Physics, volume V, Statistical physics, Part I, M., Science, 1976. 